\title{
VÖLKERRECHT UND ENTWICKLUNG
}

\author{
Von ERnst U. PetersmanN
}

Entwicklungshilfe ist in den letzten 20 Jahren neben der internationalen Handels-, Währungs-, Zahlungs- und Wettbewerbsordnung zu einem neuen Hauptgebiet der internationalen Wirtschaftsbeziehungen und des Internationalen Wirtschaftsrechts geworden. Die verwirrende Vielzahl von nationalen und internationalen Hilfsorganisationen, Präferenzsystemen (z. B. UNCTAD- und Assoziationspräferenzen), Entwicklungsstrategien (z. B. UN-Strategie vom 24. 10. 70, „Gemeinsame Ausrichtung" der Entwicklungspolitik durch den Assoziationsrat des Jaunde-Abkom= mens), redistributiven Prinzipien (z. B. Verzicht auf Meistbegünstigung und Reziprozität, Kapitalhilfe) und neuartigen Kooperationsverträgen beschleunigt dabei eine auch rechtssoziologisch interessante Weiterentwicklung des klassischen völkerrechtlichen "Koexistenzrechts" (W. Friedmann) zu einem neuen "Common Law of Mankind" (Jenks)". Parallel hierzu haben die als Folge der Dekolonisierung in den meisten internationalen Wirtschaftsorganisationen² zur Abstimmungsmehrheit gewordenen Entwicklungsländer sich in der "Gruppe der 77" (sie besteht inzwischen aus 96 Ländern) zu einem wirksamen „trade-unionism“ (Ansprenger) verbunden und wollen ihre theoretisch u. a. von G. Myrdal, R. Prebisch und J. Tinbergen fundierten Forderungen nach einer umfassenden Bevorrechtigung ihrer Export- und Entwicklungsbedürfnisse im Rahmen einer „die Fehler des Freihandelssystems korrigierenden“ (Prebisch), mehr dirigistisch,redistributiven „globalen Entwicklungsstrategie" möglichst durch eine UN- und UNCTAD-„Legislative“ durchsetzen und dadurch das aufgrund seiner historischen Entwicklung mehr an den Interessen der Industrieländer ausgerichtete Völkerrecht zum Instrument ihres wirtschaftlichen Fortschritts machen. Im folgenden soll kurz auf 3 wichtige Rechtsentwicklungen in diesem Bereich hingewiesen werden: 1) Das Zusammenwachsen traditionell getrennter Rechtsmassen zu einheitlichem „Völkerhandelsrecht“ (F. A. Mann, C. M. Schmitthoff) und das Entstehen autonomen „internationalen Rechts“ durch die Praxis des „Internationalisierens" transnationaler Darlehns-, Konzessionsund Entwicklungsverträge. 2) Das inhaltliche Aufspalten traditionell einheitlicher Weltwirtschaftsprinzipien im Rahmen der Weltwirtschaftsreform zugunsten der Dritten Welt und die dadurch entstandene Tendenz zum „Pluralismus“ im „Weltwirtschaftsrecht ${ }^{3}$ " und insbesondere zu jeweils unterschiedlichen Rechtsstandards für den Nord-Nord-Handel, den Nord-Süd-Handel und den Süd-Süd-Handel. 3) Die seit der UNCTAD I (1964) zunehmende Zahl neuer Rechtsgrundsätze, Rechtsstandards und neuartiger Rechtsquellen für die Entwicklungshilfe und die Außenwirtschaftsbeziehungen der Entwicklungsländer. \footnotetext{
1 So sind die traditionellen Vorstellungen vom Völkerrecht als the body of rules and principles of action Nations, 6. A., Oxford 1967) oder der klassische Grundsatz "any contract which is not a contract between states in their capacity as subjects of international law" is based on the municipal law of some country $^{\alpha}$ (Serbian Loans Case, PCIJ, Serie A, Nr. 20/21, S. 41) heute durch die Rechtsentwicklung überholt. 2 Ausgenommen bsd. die meist eine der finanziellen Beteiligung entsprechende "Stimmenponderierung“ anwendenden Währungs- und Weltbankinstitutionen.

3 Dieser üblich gewordene Begriff ist nur bei einer Beschräkung auf vorwiegend die GATT- und IMF-Mitglieder sinnvoll, da auf universaler Ebene keine multilateral-völkerrechtliche Weltwirtschaftsordnung besteht.
} 


\section{1. „Internationalisierte“ Verträge und „Transnationales Recht"}

Im Rahmen der Entwicklungshilfe sind zahlreiche neuartige Vertragstypen entstanden: „Economic Development Agreements“ zwischen einem Entwicklungsland und ausländischen privaten, staatlichen oder internationalen Geldgebern und Investoren ${ }^{4}$; "Joint International Business Ventures" zwischen ausländischen Gesellschaften und privaten und/oder öffentlichen Investoren im Entwicklungsland ${ }^{5}$; Darlehnsverträge internationaler Banken mit privaten, halbstaatlichen oder staatlichen Darlehnsnehmern in Entwicklungsländern, häufig ergänzt durch Garantie-, Rahmen- und Durchführungsabkommen mit dem betreffenden Entwicklungsland; staatliche Kreditaufträge an eine internationale Bank zugunsten dritter Entwicklungsländer ${ }^{6}$; neuartige Kooperations-, Konzessions-, Investitionsschutz-, Handelsabkommen und andere Geschäfts- und Entwicklungsverträge, die z. B. Rechtsstellung und Kontrollrechte ausländischen Hilfspersonals oder die Bedingungen für Ausschreibungen im Entwicklungsland regeln. Kennzeichnend für diese Verträge sind oft die gemeinsame Teilnahme von privaten, staatlichen und internationalen Vertragsparteien, das Verbinden privatrechtlicher, staats- und völkerrechtlicher Prinzipien und das auf verschiedene Arten mögliche „Internationalisieren "7 solcher Verträge, wodurch man wegen der internationalen Sachverhalte auch die Rechte und Pflichten der Vertragsparteien unabhängig vom Landesrecht regeln und durchführen will. Hierdurch ist das Rechtsproblem entstanden, welches Rechtssystem und welcher Richter für die Anwendung, Auslegung und Vollstreckung solcher „gemischter" Entwicklungsverträge zuständig sind und ob ein staatlicher Vertragspartner eine legislative Eingriffsmöglichkeit in das Vertragsverhältnis hat.

Die Frage nach dem anwendbaren Recht richtet sich zunächst nach der vertraglich vereinbarten Rechtswahl der Parteien, nach dem Rechtscharakter und „Schwerpunkt" des Vertrages (z. B. iure gestionis oder iure imperii?) sowie nach der Rechtsnatur der Vertragspartner. Beim Fehlen einer ausdrücklichen Verweisung auf eine nationale Rechtsordnung wird auf internationale Handelsgeschäfte zwischen Staaten oder Staaten und Internationalen Organisationen heute meist Völkerrecht angewendet. Bei Verträgen zwischen Staaten und Privaten besteht die Vermutung für die Anwendung des Landesrechts des teilnehmenden Staates, falls die Vertragsparteien nicht durch "Internationalisieren“ ihr Vertragsverhältnis aus dem Landesrecht herausgelöst und Völkerrecht, die „allgemein anerkannten Rechtsgrundsätze", die Prinzipien von Treu und Glauben oder allein die Lex contractus als anwendbares Recht vereinbart haben ${ }^{8}$. In der Rechtslehre ist zwar noch umstritten, ob bei international-privaten Verträgen nicht zum Schutz der schwächeren, privaten Vertragspartei die Anwendung eines nationalen Rechts zwingend ist und ob überhaupt Völkerrecht als anwendbares Recht gewählt werden kann, wenn nur eine Partei "Völkerrechtssubjekt" ist. Úberwiegend wird heute aber eine solche Rechtswahl als zulässig angesehen, da weder Völkerrecht noch Internationales

\footnotetext{
4 Vgl. Hyde, Economic Development Agreements, RCADI 1962, S. $271 \mathrm{ff}$. und ausführlich jetzt: T. J. Farer, Economic Development Agreements: A Functional Analysis, in: Columbia Journal of Transnational Law, 2/1971, S. $200 \mathrm{ff}$.

5 Vgl. Friedmann/Kalmanoff, Joint International Business Ventures, 1961.

6 Z. B. seitens der EWG-Länder an die EIB zugunsten von Griechenland.

7 Z. B. durch vertragliche Abrede, durch numbrella agreements", vgl. P. Weil, Problemes relatifs aux contrats passés entre un état et un particulier, RCADI 1969, III, S. 95 ff.; J. Käser, Darlehen der EIB Darlehen der Weltbank, Europarecht 1967, S. $289 \mathrm{ff}$.

8 Vgl. Schwarzenberger, The Principles of International Economic Law, RCADI 1966, S. 29 f.
} 
Privatrecht sie verbieten und eine solche Internationalisierung zahlreiche Vorteile haben kann: privaten Gesellschaften verschafft sie bei Konzessionsverträgen einen erhöhten Schutz gegen Enteignungen, da im Völkerrecht der Satz gilt „a state cannot rely on his own legislation to limit the scope of his international obligations"; der Vertrag hängt nicht mehr von der Landesgesetzgebung ab; das Problem der Wahl eines Landesrechts entfällt und bei Scheichtümern oder gewissen Entwicklungsländern kann die Anwendung des manchmal noch unzureichend ausgebildeten Landesrechts zu Rechtsunsicherheit führen ${ }^{9}$.

Die Darlehensverträge internationaler Banken mit privaten Darlehensnehmern in Entwicklungsländern wählen meist die Anwendung eines neutralen Landesrechts (so die EIB) oder schließen - wie im Falle der Weltbank - lediglich die Anwendung jeglichen Landesrechts aus („negative Rechtswahl“), so daß dann ebenfalls Völkerrecht (F. A. Mann, Schwarzenberger), Völkerrecht und Landesrecht im Sinne eines umfassenden „transnational law10" (Jessup, Hyde), die allgemein anerkannten Rechtsgrundsätze als Rechtssystem sui generis (McNair) oder nur die Lex contractus (Verdross) anzuwenden sind ${ }^{10}$. Die Finanzierungsabkommen des Europäischen Entwicklungsfonds schreiben für Rechtsstreitigkeiten stets die Zuständigkeit des Europäischen Gerichtshofs und für Streitigkeiten im Zusammenhang mit den vom assoziierten Empfängerland mit privaten Auslandsunternehmen abgeschlossenen Ausführungsverträgen das Vermittlungs- und Schiedsverfahren der Internationalen Handdelskammer vor. Das Anwenden der allgemein anerkannten Rechtsgrundsätze und des „transnationalen Rechts“ ermöglicht dabei auch die Anwendung privatrechtlicher Prinzipien (z. B. ungerechtfertigte Bereicherung) auf gemischte „quasi-völkerrechtliche" Verträge.

Die Gefahr zu großer Rechtsunsicherheit oder neuer „Abu-Dhabi-Entscheidungen 11“ ist im internationalen Vertrags- und Handelsrecht nicht mehr groß: die seit langem unternommene Rechtsvergleichung, die rechtsvereinheitlichenden Kodifikationsbemühungen vor allem der International Law Commission und der UN-Kommission für Internationales Handelsrecht (UNCITRAL) für ein einheitliches internationales Vertrags- und Völkerhandelsrecht, die Modellverträge der ECE, das aus den von der Internationalen Handelskammer veröffentlichten Sammlungen internationaler Handelsbräuche entstehende Welthandelsgewohnheitsrecht, der Ausbau des internationalen Schiedswesens, die Geschäftsbedingungen der internationalen Banken und Hilfsorganisationen haben auch den Abschluß internationalisierter Verträge erleichtert und das "Internationale Wirtschaftsrecht ${ }^{12}$ " sicherer gemacht. Die entwicklungspolitische Bedeutung einer „new lex mercatoria ... reflecting the interest of the entire international community" für die Handels- und Wirtschaftsentwicklung besonders auch der Entwicklungsländer wird auch in der Entschließung 2205 (XXI) der UN-Vollversammlung vom 17. 12. 1966 und in den Berichten der durch diese Entschließung eingesetzten UNCITRAL betont: "the harmonization, progressive unification and modernization of international trade law have an essential role to play in the development of countries ... eliminating diver-

9 Vgl. hierzu die Abu-Dhabi-Entscheidung in ICLQ 1/1952, S. $237 \mathrm{ff}$.

10 Vgl. Jessup, Transnational Law, 1956, S. 106: "Transnational Law . . includes what we know as public and private international law, and it includes national law, both public and private ${ }^{\alpha}$. (10a), vgl. Fn. 7 .

11 In dieser Entscheidung (vgl. Fn. 9) hatte sich der Schiedsrichter Lord Asquith wegen des Fehlens einer ausreichenden Rechtsordnung im Scheichtum Abu Dhabi auf die allgemein anerkannten Rechtsgrundsätze berufen, in concreto dann aber engl. Recht angewandt.

$12 \mathrm{Im}$ umfassenden Sinne von ${ }_{\text {Recht der internationalen Wirtschaft }}{ }^{\text {u }}$ unter Einschluß des Landesrechts, vgl. Langen, Studien zum Internationalen Wirtschaftsrecht, 1963, S. 24. 
gencies between national systems of law which formed barriers to the development of international trade13". Eine Vereinheitlichung des Wirtschaftsrechts - aller= dings auf anderer Ebene - wird auch durch die mit der EWG-Erweiterung und in geringerem Umfange teils auch mit den Assoziationsverträgen verbundene Rezeption des Europarechts bzw. Rechtsangleichung gefördert ${ }^{14}$.

\section{Außenhandel der Entwicklungsländer und „Pluralisierung“ des Internationalen Wirtschaftsrechts}

Die überwiegende Mehrheit der Entwicklungsländer ist Mitglied der wichtigsten internationalen Wirtschaftsorganisation wie IMF, GATT, Weltbank, UNCTAD, der Rohstofforganisationen und UN-Wirtschaftskommissionen. Außenhandel und internationale Finanzbeziehungen der Entwicklungsländer werden völkerrechtlich daher weitgehend durch die Satzungen und Politik dieser Organisation bestimmt, insbesondere durch die Welthandelsprinzipien des GATT (Meistbegünstigung, Reziprozität, Kontigentverbot, Inländerbehandlung, regionale Wirtschaftsintegration), die Währungsprinzipien des IMF (feste Währungsparitäten, Konvertierbarkeit, Beseitigung der Zahlungsbeschränkungen für laufende Transaktionen, Verbot diskriminierender Währungspraktiken, temporäre Zahlungsbilanzhilfen), die Finanzierungsgrundsätze der Weltbankgruppe, die Marktordnungen für Rohstoffe und Baumwollprodukte und die Entwicklungsprinzipien der UNCTAD. Das GATT und die IMF-Satzung enthalten jedoch Ausnahmebestimmungen zugunsten der Entwicklungsländer, und weder die Industrieländer noch die Entwicklungsländer halten sich stets streng an diese multilateralen Wirtschaftsgrundsätze, wobei das GATT und der IMF gegenüber den entwicklungspolitisch oft unvermeidlichen Abweichungen der Entwicklungsländer meist eine flexible Haltung einnehmen. Zusätzlich zum „Allgemeinen Zoll- und Handelsabkommen“ haben auch die Entwicklungsländer noch zahlreiche Integrations=, Handels-, Zahlungs- und Clearingabkommen abgeschlossen: einmal GATT-konforme Abkommen zur Errichtung von Zollunionen oder Freihandeiszonen, wobei der Präferenzhandel dann nur noch vom - durch Art. XXIV GATT vorgeformten - Integrationsvertrag und nicht mehr vom GATT geregelt wird; zum anderen bilatereale Handelsverträge mit Nichtmitgliedstaaten des GATT, Zollabkommen, „Kompensationsabkommen“ mit Staatshandelsländern, klassische Handels-, Freundschafts-, Schiffahrts- und Niederlassungsverträge und internationale Warenabkommen ${ }^{15}$.

Eine für die Außenwirtschaftsbeziehungen der Entwicklungsländer und die bisherige Struktur des Weltwirtschaftsrechts neuartige Rechtsentwicklung wird seit 1964 durch die zunehmende Reform der Welthandelsordnung zugunsten der Entwicklungsländer sichtbar: die sehr umfassenden und mit dem Kompetenzbereich beinah aller anderen Weltwirtschaftsorganisationen überlappenden UNCTAD-Zuständigkeiten für "Handel und Entwicklung“, das Inkrafttreten (27. 6. 66) der im Teil IV des GATT für „Handel und Entwicklung“ neu vereinbarten Rechtsprinzipien, die formelle Aufhebung der Meistbegünstigungs- und Reziprozitätspflichten der Entwicklungsländer gegenüber den Industrieländern durch Art XXXVI/8 GATT und

\footnotetext{
13 Report of the UNCITRAL on the work of its first session, UN 1968, Ziff. 22, 26.

$14 \mathrm{Das}$ Assoziationsrecht ist - ausgenommen die auf Art. $131 \mathrm{ff}$. EWGV beruhende "konstitutionelle Assoziation" - reines Völkerrecht. Zu den Vertragszielen der "Beitrittsassoziation" mit "Griechenland und der Türkei gehört jedoch auch die Rechtsangleichung. Bei der "Entwicklungsassoziation" EWG-AASM hat der Assoziationsrat am 22. 4. 1971 einheitliche Steuer- und Zollregelungen der AASM und am 30.11 .1971

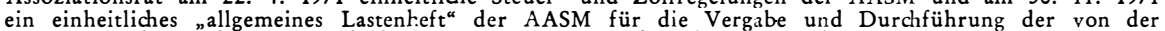
EWG im Rahmen" ihrer Kapitalhilfe finanzierten Bau- und Lieferaufträge festgelegt.

15 Vgl. G. P. Verbit, Trade Agreements for Developing Countries, New York 1969.
} 
durch das UNCTAD-Präferenzsystem sowie der wachsende Widerspruch zwischen vielen traditionellen Weltwirtschaftsprinzipien und den neuen Entwicklungsprinzipien der UNCTAD scheinen das bislang weitgehend einheitliche Recht des Welthandels in jeweils unterschiedliche Regeln für den Nord-Nord-Handel, den Nord-Süd-Handel, den Süd-Süd-Handel und den Präferenzzonenhandel aufzuspalten. Die zunehmende Einschränkung der von den Vertretern der Industrieländer als „by far the most important safeguard of order, equity and economic efficiency in international trade relations" gerühmten und von den Entwicklungsländern wegen ihrer diskriminierenden Gleichbehandlung ungleich entwickelter Länder seit langem kritisierten Meistbegünstigungspflicht des Art. I/1 GATT ist hierfür ein deutliches Beispiel: Für den Handel zwischen entwickelten GATT-Parteien gilt Art. I/1 GATT weiterhin mit dem Vorbehalt der verschiedenen GATT-Ausnahmeklauseln. Eine erste grundlegende Einschränkung von Art. I/1 und eine „zweistufige“ Meistbegünstigung ergibt sich aus der zunehmenden regionalen Wirtschaftsintegration (Art. XXIV GATT) und der völligen Umstrukturierung der Welthandelsordnung durch eine erweiterte EWG, die voraussichtlich etwa 58 Staaten zu einem euro-afrikanischen Freihandelsraum vom Nordkap bis nach Sambia zusammenfassen wird. Die dritte Abstufung der Meistbegünstigungspflicht folgt aus dem im Oktober 1970 vereinbarten und von den meisten Industrieländern (ausgenommen USA) bereits in Kraft gesetzten „allgemeinen Präferenzsystem ohne Gegenseitigkeit und Diskriminierung “ zugunsten der Entwicklungsländer und die vierte Abstufung von Art. I/1 GATT ergibt sich aus dem im „Allgemeinen Prinzip 8“ der UNCTAD I („Developing countries need not extend to developed countries preferential Areatment in operation amongst them"), aber letztlich auch schon im Teil IV GATT enthaltenen und von den Entwicklungsländern auch bereits praktizierten ${ }^{16}$ Grundsatz, daß Entwicklungsländer einander gewährte Präferenzen nicht auf Industrieländer auszudehnen brauchen und ihre Meistbegünstigungspflicht auch außerhalb von Art. XXIV GATT auf Entwicklungsländer beschränken und Industrieländer davon ausschließen können.

Ein weiteres Beispiel ist die Praxis zu Art. XXIV GATT: die angesichts der zu engen Nachfragemärkte (53 Entwicklungsländer haben weniger als 5 Mill. Einwoh= ner) unerläßlichen Integrationsabkommen zwischen Entwicklungsländern sind nach der modernen Integrationstheorie ${ }^{17}$ of nur bei erheblichen Abweichungen von Art. XXIV GATT und von der klassischen Theorie der Zollunion (Viner, Meade, Lipsey) erfolgversprechend (z. B. Beschränkung auf partielle oder sektorelle Integration) und die Praxis des GATT zur Einhaltung der Voraussetzungen des Art. XXIV GATT durch Entwicklungsländer ist dementsprechend großzügigir. Für Nord-Süd-Sonderpräferenzen (z. B. die Assoziationsabkommen der EWG) gelten dagegen wiederum andere rechtliche Beurteilungskriterien, die teils dem „Allgemeinen Prinzip 8“ der UNCTAD I (schrittweise Einfügung der Sonderpräferenzen in die Allgemeinen Präferenzen „as and when effective international measures guaranteeing at least equivalent advantages to the countries concerned come into

16 Vgl. das im Februar 1970 vom GATT gebilligte Zusatzprotokoll zum Präferenzabkommen zwischen Indien, Jugoslawien und VAR sowie die seit 1967 im GATT stattfindende Zollsenkungsrunde zwischen den Entwicklungsländern, deren Zollsenkungen aufgrund eines GATT-„waivers“ ausschließlich den 16 teilnehmenden Entwicklungsländern zugute kommen.

$17 \mathrm{Vgl.} \mathrm{z.} \mathrm{B.} \mathrm{H.} \mathrm{Bourguinat,} \mathrm{Les} \mathrm{marchés} \mathrm{communs} \mathrm{des} \mathrm{pays} \mathrm{en} \mathrm{voie} \mathrm{de} \mathrm{développement,} \mathrm{Genève,} 1968$.

18 Zulässig sind u. a. sehr lange Übergangsperioden, Liberalisierung von nur $75 \%$ des Handels und Ausschluß des Agrarhandels. 
operation") und teils dem Art. XXIV GATT (Zulässigkeit von Intcgrationsabkommen auch zwischen Ländern unterschiedlichen Entwicklungsgrads) zu entnehmen sind.

Die nicht nur unter dem Gesichtspunkt der „Handelshilfe“ notwendige Überprüfung der traditionellen Welthandelsordnung wird angesichts der in den internationalen Handelsbeziehungen eingetretenen grundlegenden Änderungen - wachsender Regionalismus, Beitritt von Staatshandelsländern und Entwicklungsländern zum GATT, zunehmende Bedeutung von multinationalen Gesellschaften (etwa $30 \%$ der Wetlexporte werden von ihnen gekauft), Präferenzen für Entwicklungsländer, verstärkte Bedeutung der nichttarifären Handelshindernisse und Zahlungsbilanzprobleme infolge des weltweit niedrigen Zollniveaus - heute auch von den Industrieländern einmütig anerkannt ${ }^{19}$.

\section{3. „Entwicklungshilferecht“}

Die rechtliche Ausgestaltung der Entwicklungshilfe hat auf verschiedenen Ebenen eine neue Rechtsentwicklung ausgelöst: im Landesrecht der Entwicklungsländer (z. B. Investitionskodices, integrationsbedingte Sonderregelungen für "Central American Integration Industries") und der Industrieländer (z. B. in der BRD das Entwicklungshilfe-Steuergesetz, die Hermes-Versicherung, die Regelung der finanziellen und technischen Hilfe, die Kabinettsbeschlüsse vom 11. 2. und 22. 12. 71 über die entwicklungspolitische Konzeption der BRD), im Organisations- und Staatengemeinschaftsrecht der internationalen Hilfs- und Wirtschaftsorganisationen, im Völkerhandelsrecht und im zwischenstaatlichen Völkerrecht. Im Hinblick auf ihren inhaltlich einheitlichen Bezug auf den Bereich der Entwicklungshilfe kann man diese sehr verschiedenartigen Rechtsnormen und Rechtsstandards vereinfachend und deskriptiv als „Entwicklungshilferecht“ zusammenfassen, ohne daß hiermit ein neuartiges Rechtssystem sui generis gemeint ist. -

C. W. Jenks wies 1967 darauf hin: „The time may have come for an attempt to deduce from the inumerable rules and agreements governing mutual aid for economic stability and growth a body of broad principles which could gradually harden into lav. In such a body of principles, the obligation to aid and the obligation to use aid wisely, for common agreed purposes . . . will necessarily be complementary to each other ${ }^{20}$." Im folgenden soll auf einige wichtige Kristallisationspunkte und mögliche Bereiche neuen völkerrechtlichen „Entwicklungshilferechts" hingewiesen werden. Diese vor allem durch die "prälegislativen“ UN- und UNCTAD-Empfehlungen ausgelöste Rechtsentwicklung unterscheidet sich vom klassischen Völkerrecht insbesondere durch die redistributiven Norminhalte, ihre sehr schnelle Entstehung und durch die Tatsache, daß viele dieser Empfehlungen noch kontrovers sind und somit entweder mangels weltweiter "opinio iuris et necessitatis“ die Schwelle zur positiven, rechtsverbindlichen Völkerrechtsnorm noch nicht überschritten haben oder zwar rechtsverbindlich (z. B. im Teil IV des GATT) vereinbart wurden, aber oft wegen ihres zu allgemein-programmatischen Inhalts keine präzisen, justiziablen Rechtspflichten begründen oder von vornherein dem Ermessen der Industrieländer anheimgestellt sind (so das auf Art. XXV/5 GATT beruhende UNCTAD-Präferenzsystem).

$19 \mathrm{Vgl}$. O. Long, Réflexions sur les mutations du commerce international, Revue du marché commun 1971, 20 C. W. Jenks, Law in the World Community, London 1967, S. 76. 
Den „Empfehlungen“ und häufig auch nur ökonomischen Standards der UNCTAD deshalb jede rechtliche Bedeutung abzusprechen, wäre dennoch ein irreführender „Juridizismus"11: der Begriff „Empfehlung“, die häufige Nichtbefolgung der von der UNCTAD empfohlenen Prinzipien, der politische Charakter und das Mehrheitsprinzip beim Zustandekommen dieser Empfehlungen sowie das Nichterwähnen von Empfehlungen als Völkerrechts-Quelle im Art. 38 Statut des IGH sind keine zwingenden Gründe für das Fehlen rechtlicher Relevanz dieser Empfehlungen, da die Völkerrechtspraxis auch per se rechtsverbindliche „Empfehlungen“ kennt (z. B. Art. 14/3 EGKSV) und Empfehlungen ausnahmsweise aufgrund besonderer Umstände einen "allgemein anerkannten Rechtsgrundsatz“ erzeugen, einen formlosen Vertrag enthalten oder als Organ- und Staatenpraxis Völkergewohnheitsrecht weiterbilden können. Beispiele für solche indirekt - durch das Hinzutreten formeller Völkerrechtsquellen - rechtsverbindlichen Empfehlungen gibt es nicht nur in der langen Praxis der Empfehlung von Rechtssätzen durch die UN-Vollversammlung ${ }^{22}$, sondern auch bereits in der UNCTAD-Praxis, wo man z. B. das ohne Gegenstimmen von 115 Staaten angenommene "Spezielle Prinzip 4" der UNCTAD I („Developing countries have the right to protect their infant industries") seit 1964 als „allgemein anerkannten Rechtsgrundsatz“ ansehen kann. Die UNCTAD-Empfehlungen können vor allem aber auch als "materielle Rechtsquelle“ die Grundlage für eine rechtliche Neuinterpretation des Völkerrechts, für einen künftigen consensus iuris über neues Völkerrecht, für einen GATT-,,waiver“ oder für den Einwand widersprüchlichen Verhaltens (wenn z. B. ein in der UNCTAD weltweit als entwicklungspolitisch notwendig anerkanntes Prinzip im GATT ignoriert wird) bilden und dadurch de teils noch hinter den "Erfordernissen der Entwicklung ${ }^{23 \text { “ der }}$ Dritten Welt hinterherhinkende Völkerrechtsentwicklung erheblich beeinflussen sowie die sonst oft mit naturrechtlichen Spekulationen verbundene Rechtsunsicherheit vermindern. Entscheidend für ihre jeweilige rechtliche Relevanz sind die Beschlußmehrheiten und näheren Umstände bei und nach der Annahme der Empfehlungen in der UNCTAD (Opinio iuris? Zustimmung der wichtigsten Welthandelsländer? Nachträgliche Anwendung?) und auch Mehrheitsbeschlüsse der heute etwa 100 Entwicklungsländer können die Völkerrechtsentwicklung dabei erheblich beeinflussen. -

\section{A. „Entwicklungsland“ und „Entwicklungshilfe“ als Rechtsbegriffe}

Die mit der zunehmenden Zahl rechtlicher Vorzugsbestimmungen zugunsten der Dritten Welt und multilateraler Entwicklungsstrategien verbundenen Auswahl- und „burden sharing“-Probleme machen eine rechtliche Bestimmung der Begriffe „Entwicklungsländer" und „Entwicklungshilfe“ unvermeidlich.

21 Hiervor - ${ }_{n}$ the mental habit of thinking primarily in legal terms and of laying all the stress on the legal framework of society without consulting the complex reality of which the economic and social facts are an important part “ - hat schon W. Röpke gewarnt, Economic Order and International Law, RCADI 1954, S. 207, 210.

22 Die einstimmig angenommene „Empfehlung betreffend Rechtsgrundsätze über die Betätigung der Staaten bei der Erforschung und Benutzung des Weltraums (1963) enthält z. B. einen formlosen Vertrag (so: Asamoah, The Legal Significance of the Declarations of the General Assembly of the UN, 1966, S. 224) oder einen allgemein anerkannten Rechtsgrundsatz (so: Zemanek, The U. N. and the law of outer space, Yearbook of world affairs, 1965, S. 209 f.), da die meisten Staatenvertreter vor der Annahme ausdrücklich die künftige Rechtsverbindlichkeit dieser Rechtsgrundsätze betonten.

$23 \mathrm{Zu}$ diesem wichtigen, besonders in der Assoziationspraxis der ElWG entwickelten Rechtsstandard vgl. z. B. Protokoll Nr. 2 des Jaunde-Abkommens von 1969 und auch O. Long, Auf dem Wege zu einer neuen Verhandlungsrunde im GATT, Europa-Archiv 7/1972, S. 234: „Hinsichtlich der Konzessionen der Entwicklungsländer ist inzwischen der Grundsatz allgemein akzeptiert, daß man von ihnen ein Entgegenkommen dann nicht erwarten darf, wenn es... ihrer eigenen Entwicklung und ihren Finanz- und Handelsbedürfnissen zuwiderliefe ${ }^{\star}$. 
Bei der Anwendung des Begriffs „Entwicklungsländer“ ist die Völkerrechtspraxis bisher flexibel - die UNO hat z. B. eine offizielle Definition vermieden - und verwendet die folgenden Auswahlmethoden:

a) Vorwiegend objektive Entwicklungskriterien liegen der 1967 von der OECD veröffentlichten Länderliste und den zwei in Art. XVIII/4 GATT enthaltenen Legaldefinitionen zugrunde, wobei das GATT bei der Anwendung von Art. XVIII die Einstufung als Entwicklungsland den betreffenden Ländern selbst überläßt. Objektive Merkmalkataloge sind zwar wegen des unzureichenden statistischen Materials über manche Entwicklungsländer und der teils unsachlichen Ergebnisse (z. B. wären die Ölscheichtümer am Pro-Kopf-Einkommen gemessen Industrieländer) nicht immer allein ausreichend, ihr Vorteil liegt aber in der objektiveren Abgrenzung vor allem auch der "border line countries“ (z. B. Israel, Hongkong, Rumänien, Bulgarien, Taiwan, die sich alle als Entwicklungsländer bezeichnen).

b) Die Methode einer kollektiven Selbstwahl wird innerhalb der UNCTAD praktiziert, so daß sich dort 5 verschiedene Gruppen von Entwicklungsländern herausgebildet haben: die 96 Länder der "Gruppe der 77“; die aus politischen Gründen aus dieser Gruppe ausgeschlossenen Länder (Israel, Taiwan und bis 1971 Kuba); die im Rahmen der UNCTAD bislang nicht als Entwicklungsländer anerkannten, 4 halbentwickelten OECD-Länder (Spanien, Griechenland, Portugal, Türkei - alle vier gelten in den DAC-Statistiken als Entwicklungsland); zwei osteuropäische Länder (Bulgarien, Rumänien) und die noch abhängigen Überseegebiete (z. B. Hongkong). Für das UNCTAD-Präferenzsystem wurde diese Auswahlmethode von den OECD-Ländern wegen der Gefahr eigennütziger oder politisch motivierter, willkürlicher Ausschließung zu Recht abgelehnt.

c) Die Auswahl durch die Geberländer ist für die bilaterale und oft auch für die multilaterale Entwicklungshilfe kennzeichnend. Für das deutsche EntwicklungshilfeSteuergesetz werden die Länderlisten durch Erlaß der Länder-Finanzministerien im Einvernehmen mit dem Bundeswirtschaftsministerium nach Stellungnahme des Auswärtigen Amtes festgelegt, und Art. 32 in Verbindung mit Protokoll Nr. 5 des LAFTA-Vertrages enthält eine einvernehmlich von den Vertragsparteien vereinbarte Aufzählung der besonders bevorzugten Mitgliedsländer.

d) Die individuelle Selbstwahl, verbunden mit einem Vorbehaltsrecht der Geberländer, ist für das UNCTAD-Präferenzsystem vereinbart worden.

e) Seit dem „Allgemeinen Prinzip 15“ der UNCTAD I ist die Notwendigkeit zusätzlicher Hilfsmaßnahmen für „besonders arme Länder“, „Entwicklungsländer mit Nachholbedarf“, „küstenferne Entwicklungsländer“, „Länder mit relativ geringer Wirtschaftsentwicklung " oder "die am wenigsten entwickelten Länder" in zahlreichen Entschließungen (z. B. der Konferenz von Algier 1967, der UNCTAD II) und Rechtsdokumenten (z. B. UN=Entwicklungsstrategie vom 24. 10. 70) allgemein anerkannt, und der UN-Ausschuß für Entwicklungsplanung hat 1971 eine Liste mit 25 dieser Länder vorgelegt, bei der von drei Abgrenzungskriterien ausgegangen wurde: BIP pro Kopf der Bevölkerung höchstens 100 Dollar, Anteil der Industrieproduktion am BIP höchstens $10 \%$ und Analphabetenquote in der

$24 \mathrm{Zu}$ der regelmäßigen Berechnung von „Nettozuflüssen“ vgl. „The Flow of Financial Resources to Less Developed Countries ${ }^{\alpha}$, OECD 1967, S. $141 \mathrm{ff}$. 
Altersgruppe ab 15 Jahren mindestens $80 \%$. Bereits vorher unterschied schon das GATT „Länder, deren Wirtschaft nur einen niedrigen Lebensstandard zuläßt und die sich im Anfangsstadium der Entwicklung befinden" (Art. XVIII/4, a) und „Länder, deren Wirtschaft sich im Entwicklungszustand befindet" (Art. XVIII/4, b) und die EWG-Kommission geht in ihrer Mitteilung an den Rat vom 29. 7. 69 über „Die Beziehungen zu den lateinamerikanischen Ländern" noch weiter und empfiehlt für die Vergabe von Technischer Hilfe eine Dreiteilung: verhältnismäßig entwickelte Länder“, „Länder auf halbem Entwicklungsstand“ und „Länder mit beginnender Entwicklung“.

Der vage Begriff „Entwicklungshilfe“ wird üblicherweise für eine Vielzahl direkter (Kapitalhilfe, Technische Hilfe, Bildungshilfe, Nahrungsmittelhilfe) oder indirekter (Handels-, Devisen- und Währungshilfen, Fördern von Auslandsinvestitionen) Hilfsmaßnahmen verwendet und wird immer umstritten bleiben, da das „reale Geschenkelement ${ }^{24 "}$ dieser unterschiedlichen Leistungen oft nicht genau zu berechnen ist und man statt des simplen „Dreiweltenmodells“ die Entwicklungshilfe ebensogut als Investition in eine gemeinsame Zukunft (neues Weltwirtschaftssystem, Weltfriede) verstehen kann. Die von der UNCTAD für die jährliche Kapitalhilfe der Industrieländer aufgestellten Zielsätze (UNCTAD I: 1\% des NSP, UNCTAD II: $1 \%$ des BSP) und die auch im Zusammenhang mit dem UNCTADPräferenzsystem schon im Hinblick auf die Gefahr von Handelsverlagerungen betonte Notwendigkeit eines gerechten „burden sharing“ machen jedoch auch hier genaue rechtliche Definitionen notwendig. Die von der UNCTAD aufgestellten Zielsätze beziehen sich auf den Begriff „financial resources“ und die hierfür von der UNCTAD festgelegte Definition ${ }^{25}$ ist wegen ihrer Addition qualitativ völlig unterschiedlicher Kapitaltransfers zu Recht kritisiert worden ${ }^{26}$, zumal genaue Berechnungen oft am unzureichenden statistischen Material (z. B. über den Kapitalrückfluß scheitern. Von den verschiedenen anderen Definitionen in der UN- und OECD-Praxis hat sich am meisten das 1969 vom DAC eingeführte Klassifizierungssystem bewährt, das jetzt "Offizielle Entwicklungshilfe“ (von staatlichen Stellen zu günstigen Bedingungen primär für Entwicklungshilfe gezahlte Mittel), „andere offizielle Auszahlungen" (staatliche Exportkredite, kommerzielle langfristige Darlehen, staatlicher Netto-Ankauf von Schuldverschreibungen multilateraler Entwicklungsbanken) und "Privaten Kapitalzufluß" (Direkt- oder Portfolioinvestitionen, private Exportkredite mit über einjähriger Laufzeit) unterscheidet und alle Angaben stets auf Nettozuflüsse bezieht ${ }^{27}$.

\section{B. Aufbringung, Konditionen und Verteilung der Entwicklungshilfe}

Die von einigen Entwicklungsländern geforderte und einer internationalen Steuerpflicht der Industrieländer gleichkommende Anerkennung eines allgemeinen Rechtsanspruchs der Entwicklungsländer auf Entwicklungshilfe und „Wiedergutmachung

$25 \mathrm{Vgl}$. Proceedings of the UNCTAD I, UN 1964, Vol. I, S. 44: “Official cash grants and grants in kind; sales of commodities against local currencies; government lending for periods exceeding one year (net of repayments of principal); grants and capital subscriptions to multilateral aid agencies, and net purchases of bonds, loans and participations from these agencies. Private capital on the basis of net long-term movements, originating, with residents of the capital-exporting countries. They are thus net of repatriation of principal, disinvestment, and retirement of long-term loans, portfolio assets and commercial debt. They are not net of reverse flows of capital originating with residents of the less developed countries, nor of investment income,

26 H. G. Johnson, Economic policies towards less developed countries London 1967, S. 118: "Such a rag bag of economi cally incomparable transactions makes a nonsense of the $1 \%$ target". Sowohl das "Aktionsprogramm von Lima“ der Gruppe der 77 (6. 11. 71) als auch der Bericht des UNCTAD-Generalsekretärs P. programm von Lima“ der Gruppe der 77 (6. 11. 71) als auch der Bericht des UNCTAD-Generalsekretärs P.
Guerrero für die UNCTAD III (vgl. TD/99 vom 15. 2. 72) schlagen eine Neudefinition der "Financial resources" insbesondere durch Bereinigung des Netto-Prinzips vor.

27 Ausführlich: OECD, AiD Review 1969, S. $239 \mathrm{ff}$. 
der Kolonialschuld" hat zwar im gegenwärtigen System der internationalen Beziehungen ebensowenig Aussicht auf Erfolg wie Versuche, solche Rechtspflichten zur Entwicklungshilfe in Art. 56 SVN, in das "Allgemeine Prinzip der guten Nachbarschaft" (vgl. Art. 74 SVN) oder in die Entschließungen der UNCTAD hineinzuinterpretieren. Im Rahmen engerer Rechtsvereinbarungen bestehen dagegen schon heute Rechtspflichten zur Entwicklungshilfe gegenüber abhängigen Úberseegebieten (Art. 132/2 EWGV, Art. 73 SVN), assoziierten Entwicklungsländern (Jaunde-Abkommen), im Rahmen multilateraler Abkommen zwischen Industrieländern (Nahrungsmittelhilfeabkommen von 1967 und 1971) oder im Rahmen anderer Vereinbarungen mit Entwicklungsländern.

Von den zahlreichen internationalen „Konditionenempfehlungen“ sei die DACEmpfehlung von 1965 erwähnt, die zwei alternative Standards aufstellt: entweder ein Anteil der Schenkungen an der öffentlichen Kapitalhilfe von $70 \%$ oder eine Ubereinstimmung mit allen drei folgenden Kriterien: ein Anteil der Schenkungen und Darlehen mit einem Zinssatz von höchstens $3 \%$ von insgesamt $81 \%$, 82\% der öffentlichen Kapitalhilfe in Form von Schenkungen und Darlehen mit einer Laufzeit von mindestens 25 Jahren und eine tilgungsfreie Zeit von durchschnittlich sieben Jahren. Die DAC-Konditionenempfehlung von 1969 ist zwar bereits günstiger als die DAC-Empfehlung von 1965, jedoch härter als die UNCTAD-Konditionenempfehlung 29 (II) und ais die Empfehlungen der Pearson-Kommission. Für die jährlichen Netto-Auszahlungen der Industrieländer an die Entwicklungsländer empfiehlt die UN-Entwicklungstrategie vom 24. 10. 70 (Ziff. 41 ff.) eine Höhe von $1 \%$ des BSP zu Marktpreisen, einen Anteil der öffentlichen Mittel von 0,7\% des BSP und u. a. eine Verringerung der Lieferbindungen. - Eine über die Bedeutung einer Empfehlung hinausgehende Rechtsverbindlichkeit dieser Entschließungen ist zwar auch künftig nicht zu erwarten. Die rechtliche Bedeutung der in diesen Empfehlungen enthaltenen "opinio necessitatis“ könnte jedoch im Zusammenhang mit den „Jahresprüfungen“ des DAC oder präziseren Vereinbarungen über ein „burden sharing " zunehmen und zeigt sich schon jetzt bei der Übernahme dieser Standards in die am 11. 2. 71 von der Bundesregierung verabschiedete „Entwicklungspolitische Konzeption der BRD für die zweite Entwicklungsdekade“ und ihrer Berücksichtigung bei der mittelfristigen Finanzplanung.

Auch für die Verteilung der Entwicklungshilfe enthalten die UN- und UNCTADEntschließungen zahlreiche Standards, die in anderen Rechtsdokumenten (Teil IV GATT, Charta von Algier) bestätigt werden: Bevorzugung der am wenigsten entwickelten Länder, Sondermaßnahmen für Entwicklungsländer ohne Zugang zum Meer, „mindestens gleichbleibende Vorteile“ für die von der schrittweisen Beseitigung der Sonderpräferenzen im Zusammenhang mit einem generellen Präferenzsystem betroffenen Entwicklungsländer, keine Diskriminierung zwischen Entwicklungsländern mit unterschiedlichen sozio-ökonomischen Systemen oder das Ziel von „stabilen, lohnenden und gerechten Preisen“ für die internationale Rohstoffpolitik. Obgleich es sich hier noch mehr um politische als um gewohnheitsrechtliche Standards handeln dürfte, kommt ihnen z. B. bei der Prüfung der Vereinbarkeit der Assoziationspräferenzen mit Art. XXIV GATT bereits eine erhebliche rechtliche Bedeutung zu, und auch die Zurückweisung der im Zusammenhang mit den UNCTAD-Präferenzen von den USA erhobenen Forderung nach vorheriger Beseitigung aller Nord-Süd-Sonder- und -Gegenpräferenzen durch die EWG-, Assoziations- und Cornmonwealth-Länder stütze sich auf die schon im „Allgemeinen Prinzip 8" der UNCTAD I enthaltenen Standards. 


\section{Ausführung der Entwicklungshilfe und Wirtschaftssouveränität der Empfänger- länder}

Für die mehr technisch-administrativen Aspekte der Durchführung der Entwicklungshilfe enthalten die Geschäftbedingungen, Verfahrensregeln, Standard-, Rahmen- und Finanzierungsabkommen der internationalen und nationalen Hilfsorganisationen zahlreiche einheitliche oder ähnliche Grundsätze (z. B. für Streitbeilegungsverfahren, die Rechtsstellung ausländischen Hilfspersonals, die Ausschreibung und Vergabe der Projektaufträge, die Zoll- und Steuerregelung für damit zusammenhängende Einfuhren, die Überwachung und spätere Unterhaltung der Projekte), ebenso wie die Assoziations-, Präferenz- und Rohstoffabkommen oder die Finanzierungsgrundsätze der regionalen Entwicklungsbanken einheitliche Rechtstechniken herausbilden (z. B. Konsultationspflichten, Sicherheiten und Kündigungsgründe bei Darlehen).

Die Rechtsstandards für den Bereich der Wirtschaftssouveränität der Empfängerländer sind dagegen noch vage und oft umstritten: die entwicklungspolitische $\mathrm{Zu}$ sammenarbeit soll auf den Prinzipien der souveränen Gleichheit, Selbstbestimmung und Nichteinmischung beruhen (Allgemeines Prinzip I der UNCTAD I); finanzielle und technische Hilfe darf nicht zur Beeinträchtigung der Souveränität der Empfängerländer benutzt werden (UN-Strategie vom 24. 10. 70, Ziff. 46) und soll frei von politischen oder militärischen Bedingungen sein (Allgemeines Prinzip 11 der UNCTAD I); dementsprechend versucht „die Bundesregierung nicht, den Partnerländern politische sowie gesellschafts- und wirtschaftspolitische Vorstellungen aufzudrängen" (Kabinettsbeschluß vom 11. 2. 71); die Außenhandelsautonomie und „Erfordernisse der Entwicklung“ der Entwicklungsländer müssen bei Freihandelszonen mit Industrieländern möglichst weitgehend geschützt werden (vgl. z. B. Art. 3, 7, 11 - 15 Jaunde=Abkommen 1969, Allgemeines Prinzip 10 der UNCTAD I); jedes Land hat das „sovereign right freely to trade with other countries, and freely to dispose of its natural resources in the interest of the economic development and well-being of its own people" (Allgemeines Prinzip 3 der UNCTAD I). Diese sehr allgemeinen Prinzipien können aber eine "neokolonialistische" Ausnutzung wirtschaftlicher Ubermacht sicher nicht verhindern und bedürfen genauerer Konkretisierungen z. B. für die Entschädigungspflicht bei binnenwirtschaftlicher Dekolonisierung und Enteignung von Rechtstiteln mit „negativen kolonialen Bezügen"28", die Zulässigkeit von Sanktionen bei entschädigungsloser Enteignung oder bei Konflikten zwischen nationaler, regionaler und globaler Entwicklungsplanung.

Die auch ohne „Entwicklungsdiplomatie“ oft unvermeidlich aus dem wirtschaftlichen Machtgefälle resultierenden, faktischen Einschränkungen der Wirtschaftssouveränität (z. B. Abhängigkeit der monokulturellen Rohstoffexportländer von den Kaffee- und Kakaoeinfuhren der westlichen Industrieländer ${ }^{29}$ ) oder die in der Entschließung 1803 (XVII) der UN-Vollversammlung enthaltene Dialektik zwischen dem grundsätzlich „inalienable right to all states freely to dispose of their natural wealth and resources in accordance with their national interest" und dem gleichzeitig anerkannten Grundsatz pacta sunt servanda

28 Vgl. Dölle, Reichert-Facilides, Zweigert, Internationalrechtliche Betrachtungen zur Dekolonisierung, 1964, S. $69 \mathrm{f}$

29 Die kaum nennenswerten Kaffeeeinfuhren des Ostblocks (z. B. Chinas) zeigen die zur "Ausbeutung durch Außenhandel“ wesentlich schlimmere Alternative der Arbeitslosigkeit "jenseits der "Ausbeutung (H. Perroy: "sous-exploitation ${ }^{\star}$ ). 
("Foreign investment agreements freely entered into by or between sovereign states shall be observed in good faith") zeigen im übrigen die Schwierigkeit einer völkerrechtlichen Regelung dieser oft mehr politischen Souveränitätsprobleme: die Industrieländer werden sich nur selten eine neue Völkerrechtsentwicklung zur Einschränkung ihrer Wettbewerbs- und Produktivitätsvorteile aufdrängen lassen (z. B. die Ausdehnung der Küstengewässer auf 200 Meilen zwecks Verdrängung der Fischfangflotten der Industrieländer) und die (seit 1951) drei Revisionen des Ol-Konsortium-Vertrags zwischen dem Iran und der National Iranian Oil Campany einerseits und dem Iranian Oil Consortium andererseits sind ein deutliches Beispiel dafür, daß die außenwirtschaftliche Abhängigkeit der Entwicklungsländer letztere zu sonst nicht eingegangenen und möglicherweise auf keiner echten Rechtsüberzeugung beruhenden Verpflichtungen zwingen kann und „Reformregierungen“ auch ohne ausreichendes Kapital für "prompte, volle und effektive“ Entschädigung zu Enteignungsmaßnahmen „in the interest of the economic development and well-being of its own people" gezwungen sein können. Der verfassungs-, satzungsoder gesellschaftsrechtlich regelmäßig bestehenden Pflicht der Geberstelle zum effektiven Einsatz ihrer Hilfe und einer völkerrechtlichen "Sozialpflichtigkeit" der Industrieländer muß eine korrespondierende Pflicht der Empfängerländer zur zweckentsprechenden Verwendung der Entwicklungshilfe und zur Unterstützung der hierfür erforderlichen Kontrollen entsprechen und die inzwischen von fast allen Entwicklungsländern erlassenen Investitionskodices dürften im übrigen die solideste Grundlage für einen Ausgleich der "natürlichen Interessengegensätze“ zwischen Gastland und künftigen Auslandsinvestoren sein. Die souveränitätseinschränkenden Auswirkungen entwicklungspolitischer Auflagen (z. B. die seitens der Weltbank von Uganda erzwungene Erhöhung der Elektrizitätsgebühren) wird man völkerrechtlich meist auch nur von Fall zu Fall beurteilen können.

\section{D. „Nord-Süd-Handel“}

Die seit der Entschließung der UN-Vollversammlung über „International Trade as the primary instrument for economic development" (1961) allgemein anerkannte Notwendigkeit einer Einbeziehung der Dritten Welt in den Kreislauf der industriellen Arbeitsteilung ("Trade not Aid“) hat während der 60er Jahre zu einem grundlegenden Wandel im Recht des Nord-Süd-Handels geführt. Wurden früher Handelspräferenzen und Diskriminierung gleichermaßen als synonyme Begriffe verwendet, so sind Präferenzen für Entwicklungsländer inzwischen zu einer von GATT und UNCTAD anerkannten Rechtstechnik für die Reform und Liberalisierung des Welthandels geworden. Neben dem grundlegenden Verzicht der Industrieländer auf Reziprozität (Art. XXXVI/8 GATT) und Meistbegünstigung (UNCTAD-Präferenzsystem) und den im vorangegangenen Abschnitt bereits erwähnten Prinzipien sind zahlreiche neue Rechtsstandards entstanden: Rechtspflicht der Industrieländer zum „standstill“ und zur Liberalisierung bei Handelshindernissen für Exporte der Entwicklungsländer (Art. XXXVII/1-3 GATT, Ziff. 33 UN-Entwicklungsstrategie); neue Nord-Süd-Präferenzen sollen nichtdiskriminierend für alle Entwicklungsländer gewährt werden, Wirtschaftsgruppierungen zwischen Industrieländern sollen für Entwicklungsländer nachteilige Handelsverlagerungen vermeiden, die Wirtschaftsintegration zwischen Entwicklungsländern soll gefördert und jede Art von Dumping unterlassen werden (Allgemeine Prinzipien 2, 8-10 und Spezielles Prinzip 9 der UNCTAD I); das Recht der Ent- 
wicklungsländer zum Schutz ihrer „infant industries“ wird anerkannt (Spezielles Prinzip 4 der UNCTAD I) und ebenso das Recht der Staaten ohne Meeresküste auf Transithandel und freien Zugang zum Meer (Anhang A. I. 2 der Schlußakte der UNCTAD I). Diese in der UNCTAD-, GATT-, Assoziations- und Völkerrechtspraxis der Entwicklungsländer entstehenden Ansätze für ein neues „Droit commercial de développement" (Kosheri) sind zwar infolge der teils noch divergierenden Handels- und Entwicklungspolitiken der großen "Welthändler" (EWG, USA, Japan) und Welthandelsorganisationen (GATT, UNCTAD, Zehnergemeinschaft + Assoziierte) oft noch kontrovers, und auch die UNCTAD hat ihre Aufgabe "to initiate action ... for the negotiation of multilateral legal instruments in the field of trade" 30 bislang nur sehr unzureichend erfüllen können ${ }^{31}$. Völkerrechtlich wichtig ist jedoch, daß die in diesen neuen Prinzipien und UNCTAD-Empfehlungen enthaltene und der weltweiten „opinio iuris“ oft vorauseilende, weltweite „opinio necessitatis“ zugunsten einer systematischen, handelspolitischen Bevorzugung der Dritten Welt die Völkerrechtspraxis der Entwicklungsländer entscheidend beeinflußt (z. B. bei der Anwendung von Art. XXIV GATT) und überall dort eine de-facto-Völkerrechtsrevision erleichtert, wo durch das Konsensprinzip die formelle Revision gewisser, die Entwicklungsbedürfnisse der neuen Staatenmehrheit der etwa 100 Entwicklungsländer vernachlässigender GATT- und IMF-Prinzipien erschwert wird. -

Die zukünftige rechtliche Bedeutung der hier aufgezeigten, entwicklungspolitischen und rechtlichen Entwicklungen im Zusammenhang mit der Entwicklungshilfe und der Weltwirtschaftsreform zugunsten der Dritten Welt läßt sich oft noch nicht abschätzen. Angesichts der zunehmenden Bedeutung des Rechts als Instrument der Entwicklungsländer und des Entstehens von „Entwicklungshilferecht" wurde 1967 in New York bereits ein "International Legal Center" gegründet, das neben der Gewährung von Technischer Hilfe im rechtlichen Bereich Grundlagenforschung über die Rechtsprobleme der Entwicklungländer und Entwicklungshilfe betreibt und diesen Rechtsfragen „in Lehre und Forschung Eingang verschaffen will 32 “.

30 Vgl. Empfehlung 1995 (XIX) der UN-Vollversammlung vom 30. 12.1964.

31 Erfolge der UNCTAD sind das Abkommen über den Transithandel der Länder ohne Meeresküste, das Zuckerabkommen 1968, das Zinnabkommen 1971 und die Errichtung der UNIDO, während das UNCTADPräferenzsystem auf keinem verbindlichen Abkommen beruht.

32 Vgl. Entwicklung und Zusammenarbeit, 2/1969, S. 10. 
The discussion of the essential point of the plan, the cooperatives, demonstrates that these cooperatives did not work profitably; further, the absolutely authoritarian organization of the cooperatives was a major factor in accounting for the fact that the farmers could not be won to participate wholeheartedly in the production.

The Tunisian experiment to establish agricultural cooperatives led to an economic disaster and was finally rejected by the population as a whole: On the one hand, the experiment had proletarized the small farmers - a situation which naturally caused their aversion to the cooperatives; on the other hand, the Tunisian landlords, who had not been expropriated, feared for their property in the event that the experiment would be extended.

The last section of the article deals with various criticisms of the Tunisian cooperatives. The authors of these critiques agree that the establishment of cooperatives in Tunisia did not lead to a transformation of the agricultural structures and, consequently, of the total social structures; they differ, however, in explaining the causes of the experiment's failure.

\section{International Law and Development Aid}

\section{By Ernst U. Petersmann}

The "widening gap", the adjustment of the still "dualistic" world economic system to the development needs of the some 100 underdeveloped countries, "trade and aid", more effective co-determination for the "Third World" in world economics, the removal of discriminatory trade practices ("equality of treatment is equitable only among equals"), the replacement of traditional "laissez faire"-principles by global and regional Development Strategies (commodity-agreements, one-way preferences etc) and, in general, more social justice on a world wide scale are among the primary concerns of international efforts, institutions and economic relations since the first "Decade of Development". The growing "International Economic Development Law" (Friedmann), the "Droit commercial de Développement" (Kosheri) as well as the UN- and UNCTAD-"legislation" are of primary importance as an instrument of development, and the development needs and non-commercial aid relations require far-reaching changes in traditional international law. The present article provides a survey of some major legal developments in this respect: the growing "Transnational Law" and the need for a "new lex mercatoria reflecting the interest of the entire international community"; the "pluralistic" tendency in International Trade Law for different rules in "North-North-Trade", "North-South-Trade" and "South-South-Trade"; some major areas of the new "Entwicklungshilferecht" like: the legal significance of the UNCTAD-recommendations, defining "underdeveloped countries" and "aid" in the many implementing and special regulations, legal standards for the distribution and carrying-through of "aid", legal problems of economic sovereignty and new standards in "NorthSouth-Trade". 